\title{
OPEN Reduced 25-hydroxyvitamin D concentration in the aqueous humor of cataract patients with open-angle glaucoma
}

\author{
Yongwun Cho ${ }^{1,6}$, Seung PilYun ${ }^{4,6}$, Woong-Sun Yoo ${ }^{1,2}$, Rock-Bum Kim ${ }^{3}$, Min-Chul Cho ${ }^{2,5,6}$ \& \\ Seong-Jae $\operatorname{Kim}^{1,2,6 \bowtie}$
}

Previous epidemiological studies have demonstrated that the lower serum concentration of vitamin $D$ was associated with elevated risk of open-angle glaucoma (OAG). However, few studies have examined the association between aqueous humor vitamin $D$ concentrations and OAG. Hence, we investigated the relationship between 25 -hydroxyvitamin $\mathrm{D}(25(\mathrm{OH}) \mathrm{D})$ concentrations in aqueous humor and OAG. We measured 25(OH)D concentrations in aqueous humor and serum of 126 patients who underwent cataract surgery. 36 were patients with OAG and 90 were control patients. The 25(OH)D concentrations were measured using Elecsys Vitamin D Total Kits with the Cobas e602 module (Roche Diagnostics, Mannheim, Germany), an electrochemiluminescence assay. Multiple linear regression analysis was performed to investigate factors associated with serum and aqueous humor 25(OH)D concentrations. Patients with OAG had significantly lower 25(OH)D concentrations in aqueous humor than control patients. Serum $25(\mathrm{OH}) \mathrm{D}$ concentrations were higher in patients with OAG than in the control, but this was not statistically significant. 25(OH)D concentrations in aqueous humor of patients with OAG were significantly associated with axial length but not with glaucoma severity, which was determined by the retinal nerve fiber layer thickness or mean deviation. Vitamin D concentrations in aqueous humor of patients with OAG were significantly lower than those in patients without OAG.

Glaucoma is a leading cause of irreversible blindness worldwide, contributing to approximately $10 \%$ of cases of legal blindness registered in the United States ${ }^{1}$. The core event in glaucoma is irreversible damage of retinal ganglion cell axons, which carry visual information from the eye to the brain, due to elevated intraocular pressure $(\mathrm{IOP})^{2}$. Depending on the mechanism of increasing IOP, glaucoma could be classified into two categories: angleclosure glaucoma (ACG) or open-angle glaucoma (OAG). Unlike ACG, which has a narrow iridocorneal angle, OAG is characterized by increased resistance in the trabecular meshwork (TM), which causes an elevation of IOP. Several studies have suggested that changes in the concentration of various molecules in aqueous humor, such as vitamin $C^{3}$, hyaluronic acid ${ }^{4}$, transforming growth factor $\beta(\text { TGF- } \beta)^{5}$, and endothelin- $1^{6}$, increase TM resistance.

Vitamin D does not exhibit biological activity until two-step hydroxylation occurs. Following the hydroxylation of vitamin D into 25-hydroxy vitamin $\mathrm{D}[25(\mathrm{OH}) \mathrm{D}$ ] in the liver, the vitamin $\mathrm{D}$ metabolite is transported to kidneys where it is converted to $1 \alpha, 25$-dihydroxyvitamin $\mathrm{D}[1 \alpha, 25(\mathrm{OH}) 2 \mathrm{D}]$, which is an active form of vitamin $\mathrm{D}^{7,8}$. The vitamin $\mathrm{D}$ status is usually evaluated by measuring $25(\mathrm{OH}) \mathrm{D}$ concentrations. Its deficiency affects bone and mineral metabolism; however, recent studies have reported that vitamin D insufficiency is associated with various systemic diseases. Adequate vitamin $\mathrm{D}$ intake can prevent diseases, such as myocardial infarction, stroke, diabetes mellitus types 1 and 2 , infectious or chronic respiratory diseases, and autoimmune diseases ${ }^{8-12}$.

\footnotetext{
${ }^{1}$ Department of Ophthalmology, Gyeongsang National University Hospital and Gyeongsang National University College of Medicine, 79 Gangnam-ro (90 Chiram-dong), Jinju-si, Gyeongsangnam-do 52727, Korea. ${ }^{2}$ Institute of Health Science, Gyeongsang National University, Jinju, Korea. ${ }^{3}$ Department of Preventive Medicine, Gyeongsang National University Hospital and Gyeongsang National University College of Medicine, Jinju, Korea. ${ }^{4}$ Department of Pharmacology and Convergence Medical Science, Institute of Health Sciences, College of Medicine, Gyeongsang National University, Jinju, Korea. ${ }^{5}$ Department of Laboratory Medicine, Gyeongsang National University Hospital and Gyeongsang National University College of Medicine, Jinju, Korea. ${ }^{6}$ These authors contributed equally: Yongwun Cho, Seung Pil Yun, Min-Chul Cho and Seong-Jae Kim. ${ }^{\square}$ email: maya12kim@naver.com
} 


\begin{tabular}{|c|c|c|c|c|}
\hline Variables & Total (\% or SD) & Control (\% or SD) & OAG (\% or SD) & $p$ value $^{*}$ \\
\hline Total & 126 & 90 & 36 & 0.32 \\
\hline Male & $72(57.14)$ & $54(60.00)$ & $18(50.00)$ & \\
\hline Female & $54(42.86)$ & $36(40.00)$ & $18(50.00)$ & \\
\hline Age (years) & $65.71 \pm 13.79$ & $66.26 \pm 14.55$ & $64.36 \pm 11.77$ & 0.23 \\
\hline Average outdoor activity time (h/day) & $2.80 \pm 2.31$ & $2.78 \pm 2.24$ & $2.83 \pm 2.50$ & 0.98 \\
\hline Mean RNFL thickness & & & $65.81 \pm 18.81$ & \\
\hline Mean visual field MD & & & $-16.4 \pm 8.67$ & \\
\hline Axial length $(\mathrm{mm})$ & $23.85(1.91)$ & $23.63(1.88)$ & $24.39(1.89)$ & $<0.01$ \\
\hline $\mathrm{ACD}(\mathrm{mm})$ & $2.75(0.61)$ & $2.77(0.63)$ & $2.71(0.58)$ & 0.71 \\
\hline $\mathrm{AqH}$ vitamin $\mathrm{D}$ concentration $(\mathrm{ng} / \mathrm{mL})$ & $10.06(5.60)$ & $11.52(5.77)$ & $6.42(2.89)$ & $<0.01$ \\
\hline Serum vitamin $\mathrm{D}$ concentration $(\mathrm{ng} / \mathrm{mL})$ & $19.01(10.42)$ & $18.12(9.55)$ & $21.22(12.18)$ & 0.27 \\
\hline BCVA (LogMAR) & $0.45(0.30)$ & $0.39(0.29)$ & $0.60(0.28)$ & $<0.01$ \\
\hline IOP $(\mathrm{mmHg})$ & $15.84(5.34)$ & $15.08(4.71)$ & $17.75(6.34)$ & 0.10 \\
\hline $\mathrm{CCT}(\mu \mathrm{m})$ & $541.2(36.60)$ & $546.4(34.78)$ & $528.1(38.25)$ & 0.03 \\
\hline $\operatorname{ECC}\left(/ \mathrm{mm}^{3}\right)$ & $2463(530.2)$ & $2446(535.6)$ & $2504(522.3)$ & 0.20 \\
\hline Cataract grade (LOCSIII) & \begin{tabular}{|l|}
$2.8 \pm 0.90$ \\
\end{tabular} & $2.9 \pm 0.97$ & $2.7 \pm 0.92$ & 0.34 \\
\hline
\end{tabular}

Table 1. Demographic and clinical characteristics of patients with open-angle glaucoma and control subjects. $O A G$ open-angle glaucoma, $S D$ standard deviation, $R N F L$ retinal nerve fiber layer, $M D$ mean deviation, $A C D$ anterior chamber depth, $A q H$ aqueous humor, $B C V A$ best-corrected visual acuity, IOP intraocular pressure, CCT central corneal thickness, ECC endothelial cell count, LOCSIII lens opacities classification system III. ${ }^{\star}$ Comparisons between patients with OAG and control subjects based on $t$-test.

Furthermore, the serum concentrations of vitamin D has been linked to the prevalence or occurrence of eye disorders, such as diabetic retinopathy, age-related macular degeneration, myopia, and dry eye syndrome ${ }^{13-16}$. In particular, several studies have demonstrated that the serum concentration of vitamin D is associated with $\mathrm{OAG}^{17-22}$.

However, most of these studies were only epidemiological investigations of the association between serum vitamin D concentrations and eye diseases; few studies have examined the association between aqueous humor vitamin $\mathrm{D}$ concentrations and eye diseases. To the best of our knowledge, no studies have reported on the relationship between the aqueous humor vitamin D concentration and glaucoma. In the present study, we measured $25(\mathrm{OH}) \mathrm{D}$ concentrations in aqueous humor of patients with cataract with or without OAG. We then performed a comparative analysis of the $25(\mathrm{OH}) \mathrm{D}$ concentrations in the two groups to determine whether there was a correlation with the presence of OAG.

\section{Results}

Total 126 patients were enrolled in this study, and 72 were men and 54 were women. The OAG group comprised 18 men and 18 women, and the control group comprised 54 men and 36 women. The duration of outdoor activity that could affect the vitamin D concentrations was $2.83 \pm 2.50 \mathrm{~h} /$ day in the OAG group and $2.78 \pm 2.24 \mathrm{~h} /$ day in the control group. The mean ages of patients in the OAG and control groups were $64.36 \pm 11.77$ and $66.26 \pm 14.55$ years, respectively. There were no significant differences in the sex, age, mean duration of outdoor activity, severity of cataract, and underlying diseases between the two groups (Table 1). In patients with glaucoma, the mean RNFL thickness measured by optical coherence tomography was $65.81 \mu \mathrm{m}$, and the mean deviation value obtained from the visual field test was $16.4 \mathrm{~dB}$. Visual acuity was significantly lower, AL was significantly higher $(p=<0.01)$, and CCT was significantly lower $(p=0.03)$ in the OAG group than in the control group. In contrast, there was no significant difference between the two groups in terms of IOP, ECC, and ACD (Table 1).

The serum $25(\mathrm{OH}) \mathrm{D}$ concentrations were $21.22 \pm 12.18 \mathrm{ng} / \mathrm{mL}$ in the OAG group and $18.12 \pm 9.55 \mathrm{ng} / \mathrm{mL}$ in the control group, which was slightly but not significantly higher in the OAG group (Table 1). However, the vitamin D concentrations in aqueous humor were $6.42 \pm 2.89 \mathrm{ng} / \mathrm{mL}$ in the OAG group and $11.52 \pm 5.77 \mathrm{ng} / \mathrm{mL}$ in the control group, and the difference between the two groups was statistically significant $(p<0.01$; Fig. 1 ; Table 1$)$.

A multivariate linear regression analysis targeting $25(\mathrm{OH}) \mathrm{D}$ concentrations in aqueous humor was conducted for all patients, and the results showed that the presence of OAG had a significant effect on the $25(\mathrm{OH}) \mathrm{D}$ concentration in aqueous humor (odds ratio [OR] 0.57, 95\% confidence interval [CI] 0.47-0.69, $p<0.01$; adjusted OR $0.64,95 \%$ CI $0.53-0.78, p<0.01$, Table 2$)$. In the same analysis, the $25(\mathrm{OH}) \mathrm{D}$ concentration in aqueous humor was significantly higher for males (OR 1.3, 95\% CI 1.07-1.57, $p=<0.01$; adjusted OR 1.27, 95\% CI 1.06-1.52, $p=<0.01$ ), whereas the concentration became statistically lower as the AL increased (OR 0.96, 95\% CI 0.91-1.00, $p=0.07$; adjusted OR $0.93,95 \%$ CI $0.88-0.99, p=0.01$ ) (Table 2). However, there was no relationship between the $25(\mathrm{OH}) \mathrm{D}$ concentration in serum or the spherical equivalent and the concentration in aqueous humor. Figure 2 shows the correlation plot between serum and aqueous $25(\mathrm{OH}) \mathrm{D}$ concentrations of all patients.

Multivariate linear regression analysis was performed in all patients. As shown in Table 3, AL was related to the $25(\mathrm{OH}) \mathrm{D}$ concentration in aqueous humor. However, there was no significant relationship between the factors related to severity of glaucoma, such as mean deviation in the visual field test or RNFL thickness, and the $25(\mathrm{OH}) \mathrm{D}$ concentration in aqueous humor (Table 3). In addition, there was no statistical significance between the 


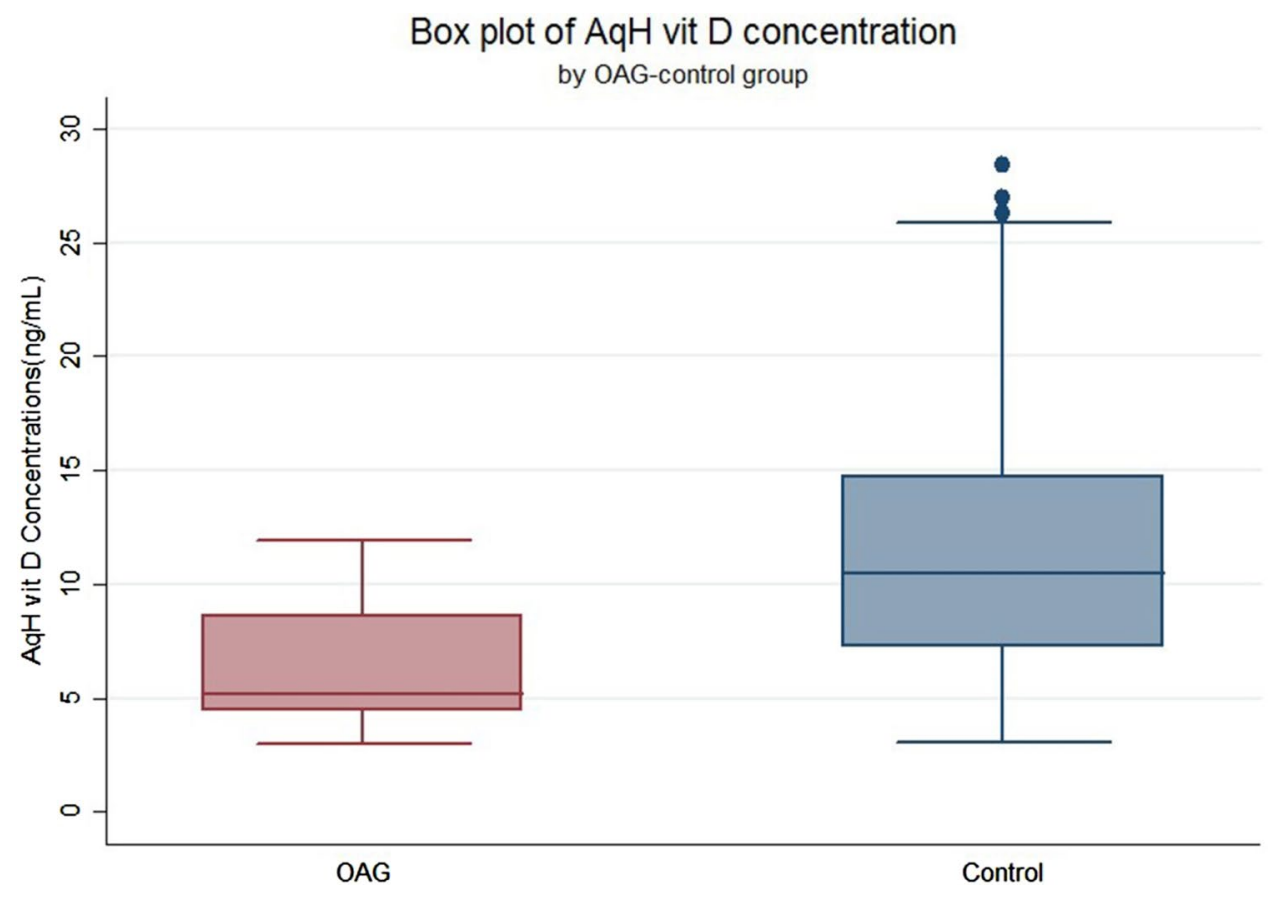

Figure 1. Concentrations of $25(\mathrm{OH}) \mathrm{D}$ in the aqueous humor of patients with open-angle glaucoma vs. control subjects. $\mathrm{OAG}=$ open-angle glaucoma.

\begin{tabular}{|l|l|c|l|c|}
\hline Variables & OR $(\mathbf{9 5} \% \mathbf{C I})$ & $\boldsymbol{p}$ value $^{*}$ & Adjusted OR (95\% CI) & $\boldsymbol{p}$ value $^{* *}$ \\
\hline OAG & $0.57(0.47-0.69)$ & $<0.000$ & $0.64(0.53-0.78)$ & $<0.01$ \\
\hline Control & 1.00 & & 1.00 & \\
\hline Serum vitamin D concentrations & $1.00(0.99-1.01)$ & 0.557 & $1.00(0.99-1.00)$ & 0.27 \\
\hline Sex & $1.3(1.07-1.57)$ & 0.007 & $1.27(1.06-1.52)$ & $<0.01$ \\
\hline Male & 1.00 & & 1.00 & \\
\hline Female & $1.00(0.99-1.01)$ & 0.847 & & \\
\hline Age (10 years) & $1.09(1.04-1.14)$ & 0.004 & & \\
\hline Average RNFL thickness & $1.00(0.98-1.02)$ & 0.836 & & 0.28 \\
\hline Visual field MD & $1.01(0.99-1.02)$ & 0.565 & & 0.01 \\
\hline IOP & $0.96(0.93-0.99)$ & 0.019 & $0.98(0.96-1.01)$ & \\
\hline Spherical equivalent & $0.96(0.91-1.00)$ & 0.076 & $0.93(0.88-0.99)$ & \\
\hline Axial length & $1.10(0.94-1.29)$ & 0.225 & & \\
\hline Anterior chamber depth & &
\end{tabular}

Table 2. Multivariate linear regression analysis of factors associated with $25(\mathrm{OH}) \mathrm{D}$ concentrations in the aqueous humor (in all patients). OAG open-angle glaucoma, RNFL retinal nerve fiber layer, $M D$ mean deviation, $I O P$ intraocular pressure, $C I$ confidence interval, $O R$ odds ratio. ${ }^{\star}$ Simple linear regression, ${ }^{* *}$ multiple linear regression.

duration of glaucoma, type of glaucoma medications, and number of drugs and aqueous $25(\mathrm{OH}) \mathrm{D}$ concentration (Table 3$)$. In the control group, no factors related to the aqueous $25(\mathrm{OH}) \mathrm{D}$ concentration were found (Table 3).

\section{Discussion}

This study confirmed that the $25(\mathrm{OH}) \mathrm{D}$ concentration in aqueous humor of patients with cataract with glaucoma was significantly lower than that in patients with cataract without glaucoma, while the presence of OAG had no significant correlation with the serum $25(\mathrm{OH}) \mathrm{D}$ concentration. The $25(\mathrm{OH}) \mathrm{D}$ concentration in aqueous humor of patients with OAG exhibited a significant correlation with AL. However, no statistical significance between the aqueous $25(\mathrm{OH}) \mathrm{D}$ concentration and glaucoma severity (RNFL thickness, MD) was detected.

The main purpose of this study was to determine whether the aqueous humor vitamin $\mathrm{D}$ concentration differs depending on the presence of glaucoma; further investigation was made into whether this difference was related to the duration of glaucoma, medications, or severity of glaucoma. Previous studies on the association between 


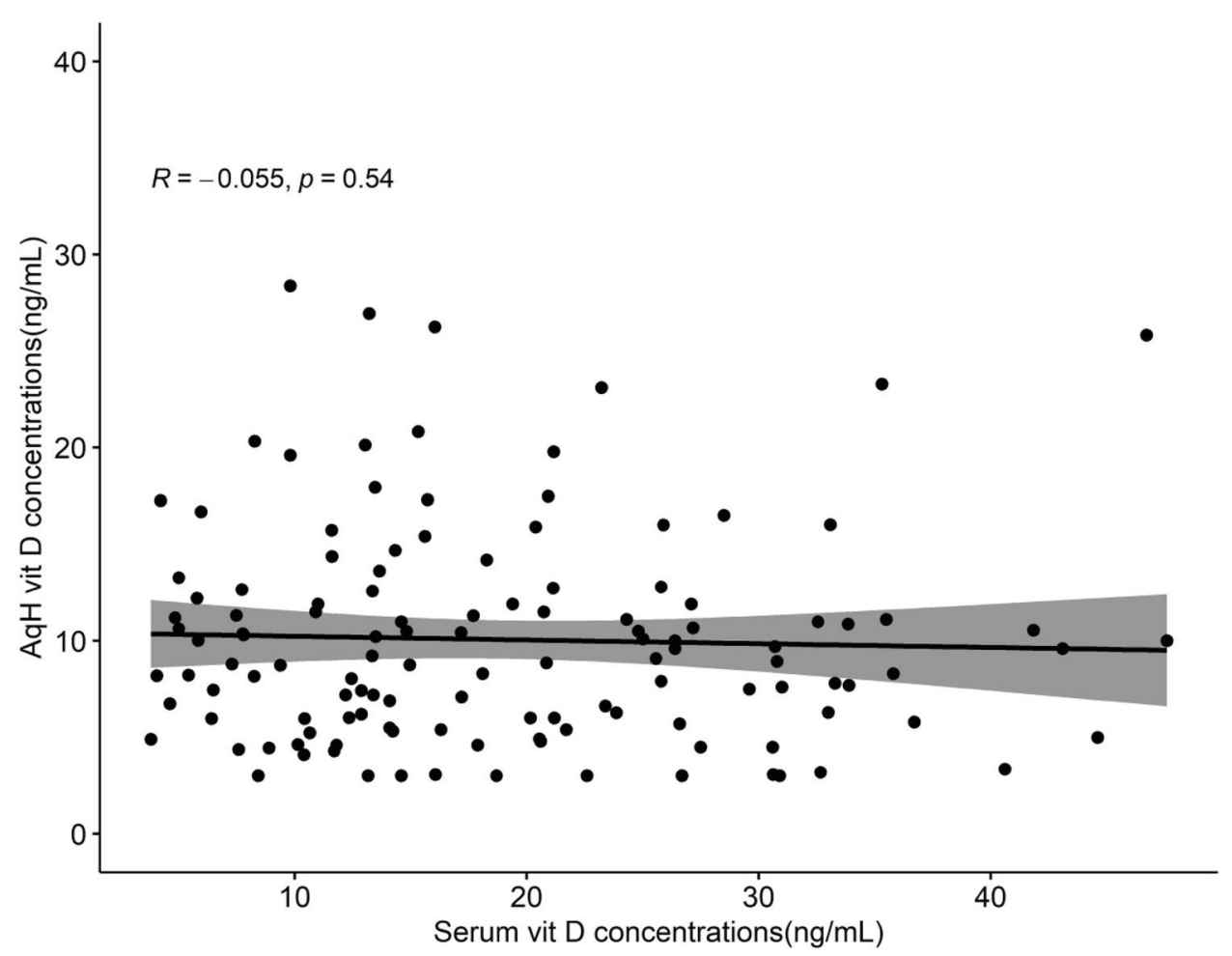

Figure 2. The correlation plot between serum and aqueous $25(\mathrm{OH}) \mathrm{D}$ levels of all patients. AqH $=$ aqueous humor; vit $\mathrm{D}=$ vitamin $\mathrm{D}$.

\begin{tabular}{|c|c|c|c|c|c|c|c|c|}
\hline \multirow[b]{2}{*}{ Variable } & \multicolumn{4}{|l|}{ Patients with OAG } & \multicolumn{4}{|c|}{ Patients without OAG } \\
\hline & OR $(95 \% \mathrm{CI})$ & $p$ value $^{*}$ & Adjusted OR (95\% CI) & $p$ value $^{* *}$ & OR $(95 \% \mathrm{CI})$ & $p$ value $^{*}$ & Adjusted OR (95\% CI) & $p$ value $^{* x}$ \\
\hline Serum vitamin D concentration & $1.00(0.99-1.02)$ & 0.60 & $1.00(0.99-1.01)$ & 0.98 & $0.99(0.99-1.01)$ & 0.83 & $1.00(0.99-1.01)$ & 0.75 \\
\hline \multicolumn{9}{|l|}{ Sex } \\
\hline Male & $1.18(0.88-1.59)$ & 0.26 & & & $1.26(1.03-1.56)$ & 0.03 & $1.23(1.00-1.52)$ & 0.05 \\
\hline Female & 1.00 & & & & 1.00 & & 1.00 & \\
\hline Age (10 years) & $1.01(0.99-1.02)$ & 0.40 & & & $0.96(0.90-1.04)$ & 0.31 & & \\
\hline Average RNFL thickness & $1.00(0.93-1.09)$ & 0.91 & & & - & - & - & - \\
\hline Visual field MD & $1.01(0.99-1.02)$ & 0.57 & & & - & - & - & - \\
\hline IOP & $1.01(0.98-1.03)$ & 0.60 & & & $1.03(1.00-1.05)$ & 0.02 & $1.02(1.00-1.04)$ & 0.06 \\
\hline Spherical equivalent & $0.96(0.92-1.01)$ & 0.11 & & & $1.00(0.9-71.02)$ & 0.84 & & \\
\hline Axial length & $0.89(0.83-0.96)$ & $<0.01$ & $0.89(0.82-0.96)$ & $<0.01$ & $1.01(0.96-1.07)$ & 0.66 & & \\
\hline Anterior chamber depth & $1.08(0.83-1.41)$ & 0.55 & & & $1.08(0.91-1.28)$ & 0.36 & & \\
\hline OAG duration (per months) & $0.99(0.97-1.01)$ & 0.41 & & & & & & \\
\hline \multicolumn{9}{|l|}{ Number of antiglaucoma medications } \\
\hline 1 & 1.00 & & & & & & & \\
\hline 2 & $0.19(0.02-1.90)$ & 0.17 & & & & & & \\
\hline 3 & $2.52(0.29-21.69)$ & 0.40 & & & & & & \\
\hline Alpha-agonist & $2.38(0.35-16.29)$ & 0.38 & & & & & & \\
\hline PG-analogs & $2.23(0.30-16.73)$ & 0.44 & & & & & & \\
\hline $\begin{array}{l}\text { Dorzolamide/timolol fixed combina- } \\
\text { tion }\end{array}$ & $0.22(0.02-2.33)$ & 0.21 & & & & & & \\
\hline $\begin{array}{l}\text { Brinzolamide/timolol fixed combina- } \\
\text { tion }\end{array}$ & $0.77(0.11-5.28)$ & 0.79 & & & & & & \\
\hline $\begin{array}{l}\text { Alpha-agonist/timolol fixed combina- } \\
\text { tion }\end{array}$ & $6.89(0.23-207.94)$ & 0.27 & & & & & & \\
\hline
\end{tabular}

Table 3. Multivariate linear regression analysis of factors associated with $25(\mathrm{OH}) \mathrm{D}$ concentrations in aqueous humor in patients with OAG and without OAG. OAG open-angle glaucoma, RNFL retinal nerve fiber layer, $M D$ mean deviation, IOP intraocular pressure, CCT central corneal thickness, ECC endothelial cell count, $C I$ confidence interval, $O R$ odds ratio, $P G$ prostaglandin. ${ }^{*}$ Simple linear regression, ${ }^{\star *}$ multiple linear regression. 
vitamin D and glaucoma generally investigated the correlation between serum concentrations and the incidence or progression of glaucoma. Among the previous studies, Yoo et al. reported that reduced serum vitamin D concentrations increased the risk for developing primary $O A G^{17}$. However, no other study has investigated the relationship between glaucoma and aqueous vitamin D concentration. Vitamin D concentrations in aqueous humor may have a stronger correlation with glaucoma than its concentrations in serum. First, aqueous vitamin $\mathrm{D}$ can have a direct effect on tissues related to the pathophysiology of glaucoma, such as the ciliary epithelium, iris, and TM. Second, there is a blood-aqueous barrier in the eye, which makes it difficult for substances present in the serum to pass directly into aqueous humor; as a result, the serum concentration does not completely reflect the concentration of aqueous humor. Reviews of previous studies on $25(\mathrm{OH}) \mathrm{D}$ indicate a lack of any significant correlation between the serum and aqueous humor vitamin $\mathrm{D}$ concentrations. Cho et al. investigated differences in the concentrations of $25(\mathrm{OH}) \mathrm{D}$ in aqueous humor and serum of senile and diabetic patients with cataract ${ }^{23}$. Thus, this study could be used as basic reference data for studies on the relationship between glaucoma and aqueous humor vitamin D concentrations.

There are few studies on the relationship between aqueous vitamin D concentration and several eye diseases. Recently, Kim et al. examined the relationship between diabetic macular edema and vitamin D concentrations in aqueous humor. They reported that diabetic macular edema was associated with high concentrations of vitamin $\mathrm{D}$ in aqueous humor and that the aqueous vitamin $\mathrm{D}$ concentration might be another indicator of diabetic macular edema severity ${ }^{24}$. In the present study, the $25(\mathrm{OH}) \mathrm{D}$ concentration in aqueous humor of patients with cataract with OAG was significantly lower than that of control patients. However, in this study, there was no correlation between the $25(\mathrm{OH}) \mathrm{D}$ concentration in aqueous humor and indirect measures of glaucoma severity, i.e., RNFL thickness and MD values.

Several experimental studies have provided evidence of the correlation between decreased vitamin D concentrations in aqueous humor and glaucoma development. The changes occurring in TM cells, such as apoptosis, compound accumulation in extracellular matrix (ECM), and cytoskeletal disruption, are primary mechanisms involved in OAG development ${ }^{25,26}$. In particular, when the concentrations of oxygen radicals in aqueous humor increase, the TGF- $\beta$-SMAD3 pathway is activated in TM cells, which results in structural changes that increase the levels of ECM proteins, such as laminin, fibronectin, and myocilin. Such changes cause fibrosis of TM cells and increase the resistance of aqueous outflow, which then lead to increased IOP. However, when $1,25-(\mathrm{OH})_{2} \mathrm{D}_{3}$ is added after treating the oxygen radicals of TM cells, VDRs are activated and block the activation of the TGF$\beta$-SMAD3 pathway, which then prevents fibrosis in TM cells ${ }^{27}$. Although structural changes in TM cells could be prevented when the concentrations of vitamin $\mathrm{D}$ in aqueous humor are high, at low concentrations, these changes in TM cells may increase the risk for glaucoma. However, to the best of our knowledge, no studies have measured vitamin D concentrations in aqueous humor of patients with glaucoma. Therefore, the findings of the present study may support this hypothesis. In addition, a previous study reported a decrease in IOP when topical $1,25-(\mathrm{OH})_{2} \mathrm{D}_{3}$ or its analog was injected in an animal experiment, and another study demonstrated an increased concentration in aqueous humor after the oral injection of vitamin $\mathrm{D}$ in rabbits. These findings indicate that vitamin D may be a potential candidate for developing a novel treatment for glaucoma.

In this study, the glaucoma group was 36 subjects, so the precision or power of the results may be low due to the small number of subjects. Further study with larger sample size might be required for more precision.

As all patients had cataract, it was difficult to eliminate the effect of cataract on the changes in vitamin D concentrations. In this study, the glaucoma group included only stable glaucoma patients who had no history of laser treatment or glaucoma surgery and who did not progress only with medications. If the study included patients with progressive visual field defect, or if the aqueous humor was collected from patients undergoing glaucoma surgery, the concentration of $25(\mathrm{OH}) \mathrm{D}$ could be measured lower than the results of this study. In addition, the correlation between the concentration of $25(\mathrm{OH}) \mathrm{D}$ in the aqueous humor and the MD values or RNFL thickness may have been statistically significant. Based on the results of this study, we plan to conduct a study by collecting a aqueous humor from patients undergoing glaucoma surgery for progressing glaucoma despite maximal antiglaucoma medication and measuring the $25(\mathrm{OH}) \mathrm{D}$ concentration. Furthermore, the effect of vitamin $\mathrm{D}$ transport on the various antiglaucoma topical medications used by patients with OAG could not be eliminated; In this study, OAG and control group showed a statistically significant difference in axial length values and the multivariate linear regression analysis revealed that there was a significant association between axial length and vitamin D concentration in aqueous humor. Previous studies demonstrated a strong relationship between vitamin $\mathrm{D}$ and myopia, so the potential role of longer axial length in determining lower concentrations of vitamin $\mathrm{D}$ in OAG group cannot be completely excluded in our study. The previous investigations that reported an association between OAG and serum vitamin D concentrations were generally large-scale studies, whereas the present study included a small number of participants. Therefore, it is challenging to make accurate judgments about serum vitamin $\mathrm{D}$ concentrations. If a large number of participants were included in this study, different results may have been obtained for serum vitamin D concentrations. Moreover, we did not survey patients regarding their dietary intake of vitamin $\mathrm{D}$, which could have had a significant influence on the test results.

In conclusion, vitamin D concentrations in aqueous humor of patients with cataract with glaucoma were significantly lower than those in patients with cataract without glaucoma. These findings suggest that future studies should explore the use of vitamin D as a candidate therapy for glaucoma treatment.

\section{Methods}

Study design. This prospective study included 126 patients who underwent uneventful cataract surgery at Gyeongsang National University Hospital between January 2017 and December 2019. This study was approved by the Gyeongsang National University Hospital Institutional Review Board (2017-01-011), and adhered to the principals of the Declaration of Helsinki. Informed consents were received from all enrolled patients. The OAG 
group included patients who were diagnosed with primary open angle glaucoma (POAG) or normal tension glaucoma (NTG). The inclusion criteria for subjects with OAG were the characteristic visual field loss, glaucomatous optic neuropathy(having neuroretinal rim narrowing or notching, retinal nerve fiber layer defects, or disc hemorrhage and disc asymmetry between both eyes $\geqq 0.2$ ), and IOP $>21 \mathrm{mmHg}$. The inclusion criteria for subjects with NTG were visual field loss, glaucomatous optic neuropathy, and IOP $\leqq 21 \mathrm{mmHg}$. Eyes with glaucomatous visual field defects were defined as (1) outside of the normal limits on the glaucoma hemifield test; (2) a pattern of standard deviation of $<5 \%$; or (3) a cluster of 3 points with probabilities of $<5 \%$ in at least 1 hemifield on the pattern deviation map, including at least 1 point with a probability of $<1 \%$ or a cluster of 2 points with a probability of $<1 \%$, as confirmed by at least 2 reliable visual field examinations. In these patients with glaucoma, glaucomatous visual field defects did not progress and IOP was stably controlled with antiglaucoma medications. The patients were also provided questionnaires to assess their occupation, regular daily activity (average time the patient was exposed to sunlight during the day), and use of vitamin D supplements. Of the patients who had undergone cataract surgery and ophthalmic examinations, we examined the medical records of patients who were receiving treatment for OAG.

Exclusion criteria. Patients who had diseases related to vitamin D, such as bone metabolism disorders or hyperparathyroidism, and patients using vitamin D supplementation were excluded. In addition, we excluded patients who had other ophthalmic diseases (vitreoretinal diseases, uveitis, and anterior segment diseases), as well as patients who underwent ophthalmological surgeries or laser treatments. In addition, patients with brunescent or hypermature cataract were excluded. Finally, cases in which the test was impossible to perform because the amount of collected aqueous humor was too low were excluded from this study.

In addition, based on a previous report that the vitamin $\mathrm{D}$ concentration in aqueous humor could be affected by the type of cataract ${ }^{23}$, we targeted only senile cataract patients, excluding those with diabetic cataract or complicated cataract.

Ophthalmic examination and cataract surgery with anterior chamber paracentesis. All procedures were conducted as described previously ${ }^{23}$. All participants provided complete medical histories and underwent ophthalmologic examination, including measurements of best-corrected visual acuity and IOP, as well as slit-lamp biomicroscopy and fundus examinations. Axial anterior chamber depth (ACD) and central corneal thickness (CCT) were measured using the Pentacam system (Oculus Inc., Wetzlar, Germany). Ocular biometry and axial length (AL) measurements were performed using the IOL Master system (IOL Master 500, Carl Zeiss Meditec, Germany). The number of corneal endothelial cells was determined using CellChek XL (Konan Medical, CA, USA). RNFL and macular thickness were measured using Spectralis Optical coherence tomography (Heidelberg Engineering, Heidelberg, Germany).

A single surgeon (S.J.K.) performed all cataract surgeries. After irrigation with $5 \%$ povidone and a balanced salt solution, approximately $0.15 \mathrm{~mL}$ of aqueous humor was collected using a 30-gauge needle through the clear cornea near the limbus. A $2.2-\mathrm{mm}$ clear corneal incision was created, and two side ports were formed. A continuous curvilinear capsulorrhexis was made using a capsulorrhexis forceps. After hydrodissection, the nucleus was phacoemulsified and the residual cortex was aspirated with an Infiniti emulsifier (Alcon Laboratories, Fort Worth, TX, USA). After filling the anterior chamber with viscoelastics, a single-piece hydrophobic acrylic IOL was inserted into the capsular bag.

Laboratory analysis. Laboratory analyses were conducted as described previously ${ }^{23}$. Immediately after collection, all aqueous humor samples were transferred to the laboratory, where their $25(\mathrm{OH}) \mathrm{D}$ concentrations were measured. In contrast, all serum samples were collected and stored at $-70{ }^{\circ} \mathrm{C}$ until analysis. The $25(\mathrm{OH}) \mathrm{D}$ concentrations were measured using Elecsys Vitamin D Total Kits with the Cobas e602 module (Roche Diagnostics, Mannheim, Germany), which is an electrochemiluminescence assay that includes the ruthenium-labeled vitamin D-binding protein, biotin-labeled vitamin $\mathrm{D}$, and streptavidin-coated microparticles. The coefficients of variation for four concentrations $(6.8,15.0,28.0$, and $67.0 \mathrm{ng} / \mathrm{ml})$ of intra- and interassay were $1.7-7.8 \%$ and $2.2-10.7 \%$, respectively.

Statistical analysis. Clinical characteristics are presented as mean and standard deviation when they were continuous variables. Categorical variables were presented as number and proportions. The characteristics of patients with cataract and OAG were compared using independent $t$-test or Fisher's exact test (in case of categorical characteristics).

To identify factors related to the vitamin D concentration in aqueous humor, univariate linear regression analysis was performed for each variable, and significant variables were included in the multivariate linear regression model and analyzed. However, the serum vitamin D concentrations were corrected by including them in the model regardless of their significance. Regression analysis was performed in all subjects (patients with OAG and cataract. Because ACH did not satisfy the normality assumption of the regression analysis, it was converted to a natural log value for the analysis and then replaced with an index and presented as a result.

Spearman's correlation analysis was performed to determine the correlation between the vitamin D concentration in aqueous humor and in serum, and the difference between the aqueous humor vitamin $\mathrm{D}$ concentration in patients with OAG and cataract was plotted.

All statistical analyses were performed using SAS version 9.4 software (SAS Institute Inc., Cary, NC, USA). A two-tailed $p$-value of $<0.05$ was considered statistically significant. 
Ethical approval. All procedures performed in studies involving human participants were in accordance with the ethical standards of the Gyeongsang National University Hospital Institutional Review Board (2017-01011), and with the 1964 Helsinki declaration and its later amendments or comparable ethical standards.

\section{Data availability}

The data used for analysis for this study are available from the corresponding author upon reasonable request.

Received: 21 May 2021; Accepted: 7 September 2021

Published online: 22 September 2021

\section{References}

1. Quigley, H. A. \& Broman, A. T. The number of people with glaucoma worldwide in 2010 and 2020. Br. J. Ophthalmol. 90, 262-267 (2006).

2. Quigley, H. A. Glaucoma. Lancet 377, 1367-1377 (2011).

3. Leite, M. T. et al. Ascorbic acid concentration is reduced in the secondary aqueous humour of glaucomatous patients. Clin. Exp. Ophthalmol. 37, 402-406 (2009).

4. Navajas, E. V. et al. Concentration of hyaluronic acid in primary open-angle glaucoma aqueous humor. Exp. Eye Res. 80, 853-857 (2005).

5. Picht, G., Welge-Luessen, U., Grehn, F. \& Lütjen-Drecoll, E. Transforming growth factor beta 2 levels in the aqueous humor in different types of glaucoma and the relation to filtering bleb development. Graefes Arch. Clin. Exp. Ophthalmol. 239, 199-207 (2001).

6. Koliakos, G. G. et al. Endothelin-1 concentration is increased in the aqueous humour of patients with exfoliation syndrome. Br. J. Ophthalmol. 88, 523-527 (2004).

7. DeLuca, H. F. Overview of general physiologic features and functions of vitamin D. Am. J. Clin. Nutr. 80(Suppl), 1689S-1696S (2004).

8. Holick, M. F. Vitamin D deficiency. N. Engl. J. Med. 357, 266-281 (2007).

9. Mitri, J. \& Pittas, A. G. Vitamin D and diabetes. Endocrinol. Metab. Clin. North Am. 43, 205-232 (2014).

10. Rai, V. \& Agrawal, D. K. Role of vitamin D in cardiovascular diseases. Endocrinol. Metab. Clin. North Am. 46, 1039-1059 (2017).

11. Illescas-Montes, R., Melguizo-Rodríguez, L., Ruiz, C. \& Costela-Ruiz, V. J. Vitamin D and autoimmune diseases. Life Sci. 233, 116744 (2019).

12. Muscogiuri, G. et al. Vitamin D and chronic diseases: The current state of the art. Arch. Toxicol. 91, 97-107 (2017).

13. Hwang, J. S., Lee, Y. P. \& Shin, Y. J. Vitamin D enhances the efficacy of topical artificial tears in patients with dry eye disease. Cornea 38, 304-310 (2019).

14. Tang, S. M. et al. Vitamin D and its pathway genes in myopia: Systematic review and meta-analysis. Br. J. Ophthalmol. 103, 8-17 (2019).

15. Layana, A. G. et al. Vitamin D and age-related macular degeneration. Nutrients 9, 1120 (2017).

16. Luo, B. A. et al. The association between vitamin D deficiency and diabetic retinopathy in type 2 diabetes: A meta-analysis of observational studies. Nutrients 9, E307 (2017).

17. Yoo, T. K., Oh, E. \& Hong, S. Is vitamin D status associated with open-angle glaucoma? A cross-sectional study from South Korea. Public Health Nutr. 17, 833-843 (2014).

18. Goncalves, A. et al. Serum vitamin D status is associated with the presence but not the severity of primary open angle glaucoma. Maturitas 81, 470-474 (2015).

19. Vuković Arar, Ž et al. Association between serum vitamin D level and glaucoma in women. Acta Clin. Croat. 55, 203-208 (2016).

20. Lv, Y. et al. Associations of vitamin D deficiency and vitamin D receptor (Cdx-2, Fok I, Bsm I and Taq I) polymorphisms with the risk of primary open-angle glaucoma. BMC Ophthalmol. 16, 116 (2016).

21. Kim, H. T. et al. The relationship between vitamin D and glaucoma: A Kangbuk Samsung health study. Korean J. Ophthalmol. 30, 426-433 (2016).

22. Li, S. et al. Lack of association between serum vitamin $B_{6}$, vitamin $B_{12}$, and vitamin D levels with different types of glaucoma: A systematic review and meta-analysis. Nutrients 9 , E636 (2017).

23. Cho, M. C. et al. Aqueous humor and serum 25-hydroxyvitamin D levels in patients with cataracts. BMC Ophthalmol. 20,6 (2020).

24. Kim, K. L. et al. Serum and aqueous humor vitamin D levels in patients with diabetic macular edema. Graefes Arch. Clin. Exp. Ophthalmol. 257, 1191-1198 (2019).

25. Tamm, E. R., Braunger, B. M. \& Fuchshofer, R. Intraocular pressure and the mechanisms involved in resistance of the aqueous humor flow in the trabecular meshwork outflow pathways. Prog. Mol. Biol. Transl. Sci. 134, 301-314 (2015).

26. Awai-Kasaoka, N. et al. Oxidative stress response signaling pathways in trabecular meshwork cells and their effects on cell viability. Mol. Vis. 19, 1332-1340 (2013).

27. Lv, Y. et al. 1a,25-Dihydroxyvitamin D3 attenuates oxidative stress-induced damage in human trabecular meshwork cells by inhibiting TGF $\beta$-SMAD3-VDR pathway. Biochem. Biophys. Res. Commun. 516, 75-81 (2019).

\section{Acknowledgements}

This work was supported by the Basic Science Research Program through the National Research Foundation of Korea (NRF) and funded by the Ministry of Education (NRF2020R1I1A3072890) and the ICT R\&D program of MSIT/IITP [2018-0-00242, Development of AI ophthalmologic diagnosis and smart treatment platform based on big data]. This research was further supported by the National Research Foundation of Korea (NRF) grant funded by the Korea government (MSIT)(No.2021R1A4A3027122) and a Grant from the Institute of Health Sciences (IHS GNU-2020-01).

\section{Author contributions}

Conception and design: S.J.K. and M.C.C. Manuscript writing/editing: Y.W.C. Statistical analysis: R.B.K. Data collection and management: W.S.Y. Data analysis: S.P.Y.

\section{Competing interests}

The authors declare no competing interests.

\section{Additional information}

Correspondence and requests for materials should be addressed to S.-J.K. 
Reprints and permissions information is available at www.nature.com/reprints.

Publisher's note Springer Nature remains neutral with regard to jurisdictional claims in published maps and institutional affiliations.

(c) (i) Open Access This article is licensed under a Creative Commons Attribution 4.0 International License, which permits use, sharing, adaptation, distribution and reproduction in any medium or format, as long as you give appropriate credit to the original author(s) and the source, provide a link to the Creative Commons licence, and indicate if changes were made. The images or other third party material in this article are included in the article's Creative Commons licence, unless indicated otherwise in a credit line to the material. If material is not included in the article's Creative Commons licence and your intended use is not permitted by statutory regulation or exceeds the permitted use, you will need to obtain permission directly from the copyright holder. To view a copy of this licence, visit http://creativecommons.org/licenses/by/4.0/.

(C) The Author(s) 2021 